\title{
Simulation for thick-target yields of transmutation reactions on radioactive targets, based on inverse kinematics
}

\author{
Shuichiro Ebata ${ }^{1, a}$, Masayuki Aikawa ${ }^{1}$, and Shotaro Imai ${ }^{2}$ \\ ${ }^{1}$ Nuclear Reaction Data Centre, Faculty of Science, Hokkaido University, Sapporo 060-0810, Japan \\ ${ }^{2}$ Institute for the Advancement of Higher Education, Hokkaido University, Sapporo 060-0817, Japan
}

\begin{abstract}
To dispose of long-lived fission products (LLFP) ejected from nuclear reactor plants is one of the most important tasks on nuclear physics and engineering. The experiments with the radiative target are limited, due to the high radioactivity and chemical property of the target. In consequence, the nuclear reaction data for LLFP are insufficient. In this work, we propose a feasible method to obtain the data for radiative targets using inverse kinematics and simulate specific systems to evaluate the thick-target yields of the nuclear transmutation reactions for LLFP.
\end{abstract}

\section{Introduction}

To reduce radioactive waste ejected from nuclear power plants is a significant topic in globe. Nuclear transmutation is a promising technology to dispose of the waste with high radioactivity. The problematic high-level radioactive waste is composed mainly of the minor actinide (MA) and long lived fission products (LLFP). For the reduction of MA, the accelerator-driven system (ADS) has been studied in global, e.g. J-PARC plans to establish the ADS test facility [1]. However, an effective procedure to reduce the LLFP has not been found yet. An essential problem for the development of LLFP transmutation is the insufficiency of the reaction data with a charged particle. Although to accumulate the data is strongly demanded, the experiments with the LLFP target are very few due to the several difficulties which are the high radioactivity and the chemical instability.

Due to the recent progress of the radioactive isotope beam technique, we can utilize LLFP nuclei as the beam. The experiments to access the data of LLFP reactions by the inverse kinematics can be namely performed, while avoiding the radioactive target [2]. When the lump of radioactive waste is assumed, it needs huge effort to obtain many data in a wide energy region. Hence we consider the integration quantity composed by microscopic values and a target matter property: a thick-target yield (TTY). The TTY is a macroscopic value which is defined with corresponding to the reaction, and is composed of the target properties such as the atomic weight $A\left(\mathrm{~g} \mathrm{~mol}^{-1}\right)$, the density $\rho\left(\mathrm{g} \mathrm{cm}^{-3}\right)$ and also the cross section $\sigma\left(\mathrm{cm}^{2}\right)$. If the TTY is obtained microscopically, the excitation function of the reaction cross section is needed. Therefore, we suggest the method to estimate the TTY of the transmutation reaction from the inverse kinematics, without measurements of microscopic values.

In this paper, we introduce briefly our method and its applications to show the availability of the method. Finally, we summarize the contents and mention the feasibility for the LLFP reaction data.

ae-mail: ebata@nucl.sci.hokudai.ac.jp 


\section{Method}

Most fundamental definition of reaction probability $Y$ can be written as a ratio between the numbers of reacted and un-reacted particle. The TTY is equivalent to this $Y$ in the case of a thick target. The differential TTY $d Y$ within an infinitesimal thickness $d x(\mathrm{~cm})$ can be described as

$$
d Y=\sigma \frac{N_{\mathrm{A}} \rho}{A_{T}} d x,
$$

where $N_{\mathrm{A}}\left(\mathrm{mol}^{-1}\right)$ is the Avogadro constant, $A_{T}\left(\mathrm{~g} \mathrm{~mol}^{-1}\right)$ and $\rho\left(\mathrm{g} \mathrm{cm}^{-3}\right)$ are the atomic weight and density of the target. Here we introduce the stopping power $S(\varepsilon)=-\frac{A_{P} d \epsilon}{d(\rho x)}\left(\mathrm{MeV} \mathrm{g}^{-1} \mathrm{~cm}^{2}\right)$ where $A_{P}$ is the projectile atomic weight and energy $\varepsilon=E / A_{P}$ is represented in the energy per nucleon. The TTY at the incident energy $\varepsilon_{\text {in }}(\mathrm{MeV} /$ nucleon), can be obtained by integrating $d Y$ for the projectile energy $\varepsilon$ in the target:

$$
Y\left(\varepsilon_{\text {in }}\right)=\frac{N_{\mathrm{A}} A_{P}}{A_{T}} \int_{0}^{\varepsilon_{\text {in }}} \frac{\sigma(\varepsilon)}{S(\varepsilon)} d \varepsilon .
$$

The lower limit of energy integration of Eq. (2) corresponds to zero, i.e., the projectile is stopped inside the target. The form of the TTY does not change in the inverse kinematics although $A_{P}$ and $A_{T}$ are exchanged, and the $S(\varepsilon)$ is changed to that of the inverse kinematics system.

Here we consider the conversion between the TTYs of the original reaction and of its inverse kinematics. The variable defined in the original reaction ("forward") is denoted as $Y_{\text {for }}$, and for its "inverse" kinematics as $Y_{\text {inv. }}$. The notations $P$ and $T$ for the projectile and target are defined as those of the original reaction. In these notations, a ratio $R(\varepsilon)$ between the differential yields $d Y_{\text {for }}$ and $d Y_{\text {inv }}$ can be expressed as

$$
R(\varepsilon)=\frac{d Y_{\mathrm{for}}}{d Y_{\mathrm{inv}}}=\frac{A_{P}^{2}}{A_{T}^{2}} \frac{S_{\mathrm{inv}}(\varepsilon)}{S_{\mathrm{for}}(\varepsilon)},
$$

because the cross section is common among the "forward" and "inverse" reactions. If we can obtain the $Y_{\text {inv }}$ and calculate the stopping powers at each projectile energy $\varepsilon$, the $Y_{\text {for }}$ can be estimated without measuring the cross sections [3]. Indeed the stopping power can be calculated accurately by the present simulation code e.g. SRIM2008 (The Stopping power and Range of Ions in Matter) [4].

\section{Result}

In this section, we show two applications for the availability of the method with the SRIM code, and for a more specific system to access the LLFP data.

\section{$3.1{ }^{\text {nat }} \mathrm{Cu}+{ }^{12} \mathrm{C}$ reaction system}

The reaction ${ }^{\text {nat }} \mathrm{Cu}\left({ }^{12} \mathrm{C}, \mathrm{X}\right){ }^{24} \mathrm{Na}$ is considered here because there are some cumulative cross section data with respect to the energy [5]. More detail data in the low energy region is necessary, thus we prepare the excitation function of the cross section, $\sigma(\varepsilon)$, from the spline interpolation of the data, shown as Fig.1 (i). The lowest energy corresponds to the roughly estimated Coulomb barrier $U_{C}$ of this system: $U_{C}=e^{2} Z_{T} Z_{P} / 1.2\left(A_{T}^{1 / 3}+A_{P}^{1 / 3}\right)$.

We estimate the TTYs using the $\sigma(\varepsilon)$ and the stopping powers $S_{\text {for }}(\varepsilon), S_{\text {inv }}(\varepsilon)$ which are calculated by SRIM 2008. The ratio $R(\varepsilon)$ is shown in Fig.1 (ii). The $Y_{\text {for }}$ and $Y_{\text {inv }}$ at $\varepsilon_{\text {in }}=40$ and $100 \mathrm{MeV} /$ nucleon are shown in the Table 1 . We can see a plateau behaviour of $R(\varepsilon)$ over $\varepsilon_{\text {in }}=50 \mathrm{MeV} /$ nucleon. The plateau of $R$ appears generally in the high energy region although the conversion energy point depends 
on the proton numbers of the reaction system, because the behaviour of a stopping power is governed by the Coulomb force. The rise of $R(\varepsilon)$ has a strong dependence on the difference between $Z_{T}$ and $Z_{P}$ [3]. Therefore we propose a more convenient conversion relation:

$$
Y_{\text {for }}\left(\varepsilon_{\text {in }}\right) \simeq \tilde{R} Y_{\text {inv }}\left(\varepsilon_{\text {in }}\right)
$$

where $\tilde{R}$ is constant value. In this example $\tilde{R}=1.1$ is chosen and the TTY $Y^{\mathrm{R}}$ estimated by Eq. (4) is shown also in Table 1. The convenient linear relation Eq. (4) well works at both 40 and 100 $\mathrm{MeV} /$ nucleon. If we can obtain the energy dependence of TTY in the inverse kinematics system, we can completely calculate the TTY of the original reaction theoretically. Even when we could get the data $Y_{\text {inv }}$ at only one energy point, we will estimate an approximate $Y_{\text {for }}$ if we use the Eq. (4).

Table 1. The TTYs of ${ }^{\text {nat }} \mathrm{Cu}+{ }^{12} \mathrm{C}$ reaction with $\varepsilon_{\text {in }}=40$ and $100(\mathrm{MeV} /$ nucleon $)$ are listed. $Y^{\mathrm{D}}$ and $Y^{\mathrm{R}=1.1}$ denote the values calculated by Eq. (2) and evaluated by Eq. (4) with $\tilde{R}=1.1$, respectively.

\begin{tabular}{c|ccc}
$\varepsilon_{\text {in }}(\mathrm{MeV} /$ nucleon $)$ & $Y_{\text {for }}^{\mathrm{D}}\left(\varepsilon_{\text {in }}\right)$ & $Y_{\text {inv }}^{\mathrm{D}}\left(\varepsilon_{\text {in }}\right)$ & $Y_{\text {for }}^{\mathrm{R}=1.1}\left(\varepsilon_{\text {in }}\right)$ \\
\hline \hline 40 & $0.91 \times 10^{-5}$ & $0.86 \times 10^{-5}$ & $0.94 \times 10^{-5}$ \\
100 & $0.114 \times 10^{-3}$ & $0.103 \times 10^{-3}$ & $0.113 \times 10^{-3}$ \\
\hline
\end{tabular}

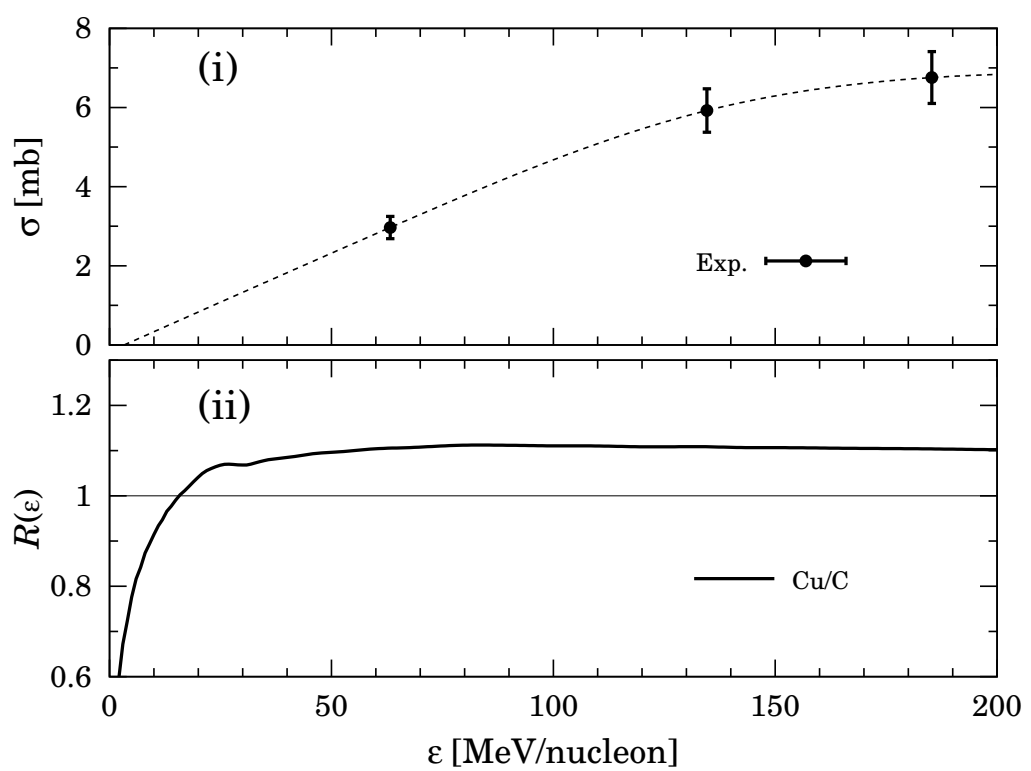

Figure 1. (i) Cross section with respect to $\varepsilon$ of the ${ }^{\text {nat }} \mathrm{Cu}\left({ }^{12} \mathrm{C}, \mathrm{X}\right){ }^{24} \mathrm{Na}$ with experimental data [5], and (ii) the evaluated ratio $R(\varepsilon)$ by SRIM 2008 [4]. 


\subsection{Application to the LLFP ${ }^{135} \mathrm{Cs}$}

${ }^{135} \mathrm{Cs}$ is one of the most problematic LLFP, which has the half-life over two million years. We simulate the transmutation reaction of ${ }^{135} \mathrm{Cs}$ bombarded by ${ }^{12} \mathrm{C}$, through their inverse kinematics. For the simulation, we utilize the PHITS (Particle and Heavy Ion Transport code System) which is a general purpose Monte Carlo particle transport simulator developed under the collaboration among JAEA, RIST, KEK and several other institutes [6]. These simulations for TTYs of ${ }^{12} \mathrm{C}\left({ }^{135} \mathrm{Cs}, \mathrm{X}\right){ }^{A^{\prime}} \mathrm{Cs}$ at several energies as the inverse kinematics of ${ }^{135} \mathrm{Cs}\left({ }^{12} \mathrm{C}, \mathrm{X}\right){ }^{A^{\prime}} \mathrm{Cs}$ reaction, are performed by the PHITS which includes an empirical formula to generate reaction cross sections.

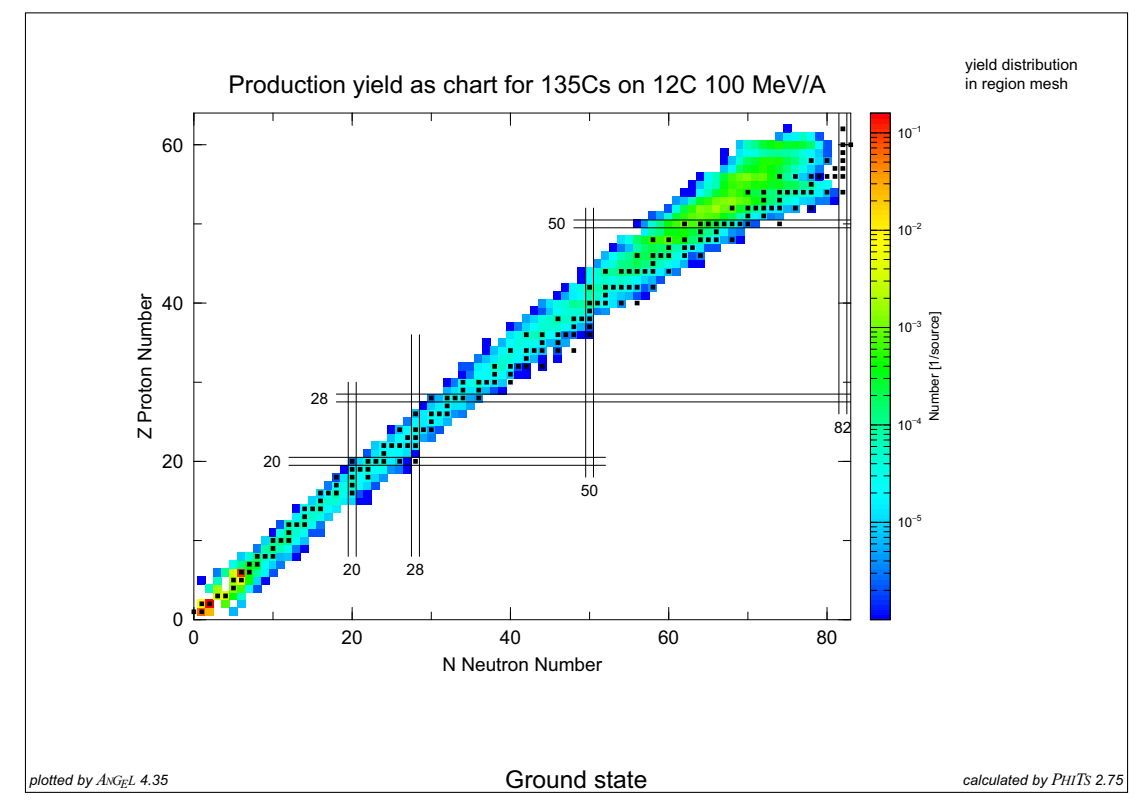

Figure 2. The distribution of fragments for ${ }^{135} \mathrm{Cs}+{ }^{12} \mathrm{C}$ reaction simulated by PHITS 2.75 .

Table 2. The PHITS condition for ${ }^{12} \mathrm{C}\left({ }^{135} \mathrm{Cs}, \mathrm{X}\right){ }^{A^{\prime}} \mathrm{Cs}$

\begin{tabular}{l|l}
\hline \hline Beam intensity & $1000 \mathrm{pps}$ \\
Irradiation time & $1000 \mathrm{~s}$ \\
${ }^{12} \mathrm{C}$ target thickness & $2.0 \mathrm{~cm}$ \\
\hline Beam energy region & $20-100 \mathrm{MeV} /$ nucleon \\
\hline \hline
\end{tabular}

The reaction induced ${ }^{135} \mathrm{Cs}$ is equivalent to the objective transmutation reaction. Figure 2 shows the distribution of fragments in the PHITS simulation at $100 \mathrm{MeV} /$ nucleon under the conditions listed in Table 2. To evaluate the TTY of ${ }^{135} \mathrm{Cs}\left({ }^{12} \mathrm{C}, \mathrm{X}\right){ }^{A^{\prime}} \mathrm{Cs}$ reaction with $A^{\prime}(\neq 135)$, we sum up the production yields of the reaction in the inverse kinematics: ${ }^{12} \mathrm{C}\left({ }^{135} \mathrm{Cs}, \mathrm{X}\right){ }^{A^{\prime}} \mathrm{Cs}$. Although the evaluated TTY does not reach that for all reaction yields, this TTY might be the lower limit of the transmutation reaction. These TTY at each energy from 20 to $100 \mathrm{MeV} /$ nucleon are displayed in Fig.3 as filled circle 
symbols. The stopping power ratio of the system is calculated by SRIM 2008, which is displayed in Fig. 3 with star symbols. Furthermore the TTY of the original reaction is evaluated using Eq. (4) with the constant stopping power ratio $\tilde{R}=1.06$, which is plotted as open circle.

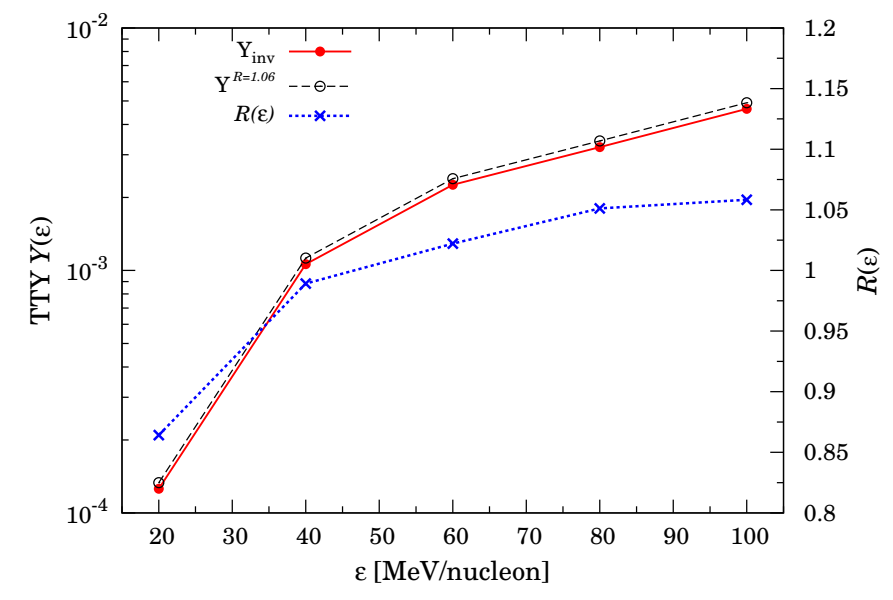

Figure 3. The TTY of ${ }^{12} \mathrm{C}\left({ }^{135} \mathrm{Cs}, \mathrm{X}\right){ }^{A^{\prime}} \mathrm{Cs}$ (solid circles), the evaluated TTY $Y^{\mathrm{R}}$ by Eq. (4) with $\tilde{R}=1.06$ (open circles), and the $R(\varepsilon)$ (stars), are shown.

\section{Conclusion}

To accumulate the data for the transmutation of LLFP, the recent progress of radioactive isotope beam facility is essential. We reported the conversion method assuming the inverse kinematics using a radioactive beam, to obtain the TTY of the reaction with a radioactive target.

When we apply the method to ${ }^{\text {nat }} \mathrm{Cu}+{ }^{12} \mathrm{C}$, the more convenient conversion relation is found, which is to replace the energy dependent ratio with the constant value. The ratio between differential TTYs $\left(d Y_{\text {for }}, d Y_{\text {inv }}\right)$ which is governed by the stopping powers decided with the combination of target and projectile, has the saturated behaviour in a high energy region. The linear relation between $Y_{\text {for }}$ and $Y_{\text {inv }}$ is justified when the reaction cross section in the low energy has only minor contribution to the TTY. We simulate also the ${ }^{135} \mathrm{Cs}$ bombarding on ${ }^{12} \mathrm{C}$ reaction with helps of PHITS and SRIM to estimate the TTY of the LLFP transmutation reaction. The TTYs of breaking up ${ }^{135} \mathrm{Cs}$ to ${ }^{A^{\prime}} \mathrm{Cs}$ by ${ }^{12} \mathrm{C}$ are estimated. Although the stopping power ratio converge to about 1.06 in this system, the tendency of the ratio is gentler up to the convergence than the previous example.

In future work, we will apply our method to more specific LLFP subjects and analyse the second particle effects for yields in the Monte Carlo simulation. Although for some LLFP data our method is effective, it has not been finished for other LLFP, yet. We should develop the extended procedure to gather the more accurate data for the transmutation technology.

\section{Acknowledge}

This works was funded by ImPACT Program of Council for Science, Technology and Innovation (Cabinet Office, Government of Japan). 


\section{References}

[1] http://j-parc.jp/Transmutation/en/index.html

[2] H. Wang et al., http://dx.doi.org/10.1016/j.physletb.2015.12.078

[3] M. Aikawa, S. Ebata and S. Imai, Nucl. Instr. Meth. B 353, 1 (2015)

[4] J. F. Ziegler, J. P. Biersack, M. D. Ziegler, SRIM: the Stopping and Range of Ions in Matter, http://www.srim.org, Nucl. Instr. Meth. B 268, 1818 (2010)

[5] H. Yashima et al., Phys. Rev. C66, 044607 (2002)

[6] T. Saito, et al., J. Nucl. Sci. Technol. 50:9, 913-923 (2013). 